\title{
miR-199a decreases Neuritin expression involved in the development of Alzheimer's disease in APP/PS1 mice
}

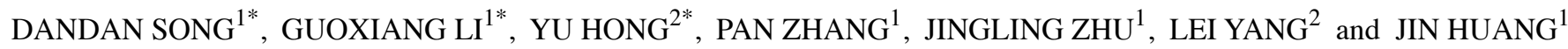 \\ ${ }^{1}$ The Key Laboratory of Xinjiang Endemic and Ethnic Diseases and Department of Biochemistry, \\ Shihezi University School of Medicine, Shihezi, Xinjiang 832002; ${ }^{2}$ Department of Prevention Medicine, \\ School of Medicine, Hangzhou Normal University, Hangzhou, Zhejiang 310036, P.R. China
}

Received December 3, 2019; Accepted April 23, 2020

DOI: $10.3892 /$ ijmm.2020.4602

\begin{abstract}
Neuritin plays an important role in neural development and plasticity. A recent study demonstrated that increasing Neuritin levels attenuated synaptic damage in mice with Alzheimer's disease (AD), which exhibit a decreased Neuritin expression. However, it remains unclear as to whether Neuritin expression is regulated by microRNAs (miRNAs or miRs) in AD. In the present study, it was found that miR-199a decreased Neuritin expression and was therefore involved in the development of AD. Subsequently, differentially expressed miRNAs in AD from datasets and the literature were recruited, and those that could bind Neuritin were predicted using bioinformatics analysis. The present study then focused on the candidate miRNAs that were highly associated with Neuritin and were upregulated in AD. The expression patterns of the candidate miRNAs and Neuritin in the hippocampus and cortex of APP/PS1 (AD model) mice at different stages were then detected and analyzed. It was found that miR-199a expression was significantly increased in the early stages of AD and was negatively associated with Neuritin expression. Furthermore, it was revealed that the decreased Neuritin expression was due to the direct targeting of the Neuritin 3'-UTR by miR-199a. Finally, the association between the spatial memory capacity of APP/PS1 mice and the changes in miR-199a and Neuritin expression protein was investigated. On the whole, the data of the present study suggest that miR-199a is involved in the development of AD by regulating Neuritin expression.
\end{abstract}

Correspondence to: Professor Jin Huang, The Key Laboratory of Xinjiang Endemic and Ethnic Diseases and Department of Biochemistry, Shihezi University School of Medicine, District 32, Shihezi, Xinjiang 832002, P.R. China

E-mail: huangjin623@163.com

${ }^{*}$ Contributed equally

Key words: miR-199a, Neuritin, Alzheimer's disease, APP/PS1 mice, hippocampus, bioinformatics

\section{Introduction}

Neuritin (or Nrn1), also known as candidate plasticity gene 15 (CPG15) $(1,2)$, is a neurotrophin that is involved in neural development and neuroplasticity (3). Neuritin promotes neurite growth and synaptogenesis in hippocampal and cortical neurons $(4,5)$, and is related to the recovery from nerve injury and learning memory (6-8). It has further been found that recombinant Neuritin (9) improves nerve regeneration following acute spinal cord injury (10) and sciatic nerve injury in rats (11).

$\mathrm{AD}$ is a chronic nervous system degenerative diseases characterized by progressive cognitive and memory impairment (12). Importantly, recent studies have demonstrated that Neuritin expression is decreased in mice with Alzheimer's disease (AD), and that Neuritin attenuates cognitive function impairments $(13,14)$. Given the role of Neuritin in AD, it may thus be a novel target for the treatment of $\mathrm{AD}$; however, the mechanisms underlying the downregulation of Neuritin expression in AD remain unclear.

Studies have demonstrated that $>33 \%$ of human genes are modulated by microRNAs (miRNAs or miRNAs) (15-17). It has also been reported that miRNAs regulate the spatiotemporal changes in Neuritin expression $(18,19)$. Therefore, it was hypothesized that specific miRNAs regulate Neuritin expression in $\mathrm{AD}$ and therefore are involved in the development of AD.

In the present study, the potent analytical and predictive capabilities of bioinformatics (20) were utilized to focus on candidate miRNAs that may regulate Neuritin expression in AD. The association between these candidate miRNAs and Neuritin expression in mice with AD was then confirmed. In addition, the mechanisms responsible for the regulatory effects of miR-199a on Neuritin expression were investigated. The associations between the memory capacities of APP/PS1 mice and altered miR-199a and Neuritin levels were also investigated in an aim to elucidate the role of miR-199a in AD and its regulatory effects on Neuritin.

\section{Materials and methods}

Analysis of microarray expression profiling data. To search for miRNA expression profiling data in AD from the Gene 
Expression Omnibus database (www.ncbi.nlm.nih.gov/geo), 'Alzheimer's disease' and 'microRNA' were used as indexes to obtain miRNA datasets. The selection criteria for microarray datasets of samples were those that could extract the original data from $\mathrm{AD}$ and normal control (NC) groups using $\mathrm{R}$ language, excluding epigenetic data. A total of 5 datasets of AD (GSE48552, GSE46579, GSE46131, GSE16759 and GSE48028), including 67 AD samples and 38 NC samples were selected for further analysis. Differentially expressed miRNAs (DEmiRNAs) were screened by a matrix of miRNA expression levels in the Bioconductor LIMMA R package. The screening threshold of DEmiRNAs was $\mathrm{P}<0.05$, Ifold changel $>1$. Additionally, literature with all the miRNA expression profiles of $\operatorname{Tg} 2576$ mice was selected for the analysis of DEmiRNAs (21).

Screening and analyzing of the candidate miRNAs. In total, 9 bioinformatics algorithms including miRWalk (http://mirwalk.umm.uni-heidelberg.de/), miRNAMap (http://mirnamap.mbc.nctu.edu.tw/), miRDB (http:// mirdb.org/), miRtarBase (http://mirtarbase.mbc.nctu. edu.tw/php/index.php), miRNApath (http://snf-515788. vm.okeanos.grnet.gr/), PicTar5 (https://pictar.mdc-berlin.de/), starBase (http://starbase.sysu.edu.cn/starbase2/), TargetScan (http://www.targetscan.org/mmu_72/) and miRanda (http://www.microrna.org/microrna/home.do), were used to predict the miRNAs that target Neuritin (or Nrn1). Moreover, an online Venn diagram software (http://bioinformatics.psb.ugent. be/webtools/Venn/) was used to screen miRNAs that were both differentially expressed in AD and that target Neuritin. Finally, TargetScan was used to predict the differentially expressed genes (DEGs) downstream of the candidate miRNAs that target Nrn1 (cumulative weighted context ++ score $<-0.1$ ). A Gene Ontology (GO) analysis was then performed using the online software DAVID (https://david.ncifcrf.gov/) to analyze the function of the targets of the candidate miRNAs, with FDR $<0.05$ used to define statistical significance.

Transgenic mice with AD. APPSWE, PSEN1dE9 (APP/PS1) transgenic mice and matched wild-type (WT) B6C3 mice were purchased from the Animal Model Center of Nanjing University. A total of $20 \mathrm{APP} / \mathrm{PS} 1$ mice and $20 \mathrm{WT}$ mice were housed under SPF conditions, $18-26^{\circ} \mathrm{C}$ with $40-70 \%$ humidity under a 12:12 h light: Dark cycle, with free access to food and water. All experimental procedures were approved by the Animal Ethics Committee of Hangzhou Normal University.

$R N A$ isolation and $R T-q P C R$. Total RNA was isolated from hippocampal and cortical tissues using the SanPrep Column microRNA Extraction kit (Sangon Biotech Co., Ltd.), and cDNA was synthesized by reverse transcription using Revert Aid Premium Reverse Transcriptase (Thermo Fisher Scientific, Inc.). Subsequently, 2X SG Fast qPCR Master Mix (Sangon Biotech Co., Ltd.) was used to amplify the 10 candidate miRNAs. The PCR thermocycling conditions of the 10 candidate miRNAs were as follows: $95^{\circ} \mathrm{C}$ for $3 \mathrm{~min}$, followed by 35 cycles at $94^{\circ} \mathrm{C}$ for $30 \mathrm{sec}, 57^{\circ} \mathrm{C}$ for $30 \mathrm{sec}$, and $72^{\circ} \mathrm{C}$ for $30 \mathrm{sec}$, ending at $72^{\circ} \mathrm{C}$ for $8 \mathrm{~min}$. The miRNA expression levels were normalized to those of U6 small nuclear RNA. Copy numbers of the 10 miRNAs were obtained using the standard curve of RT-PCR. To detect Nrn1 mRNA levels, total RNA was isolated from cortical and hippocampal tissue samples using TRIzol reagent (Invitrogen; Thermo Fisher Scientific, Inc.). Equivalent amounts of RNA from each sample were used for cDNA synthesis using the FastKing gDNA Dispelling RT SuperMix kit [Tiangen Biotech (Beijing) Co., Ltd.]. Subsequently, the cDNAs were used for qPCR analysis. The thermocycling conditions of Nrn1 mRNA are $95^{\circ} \mathrm{C}$ for $15 \mathrm{~min}$, followed by 40 cycles at $95^{\circ} \mathrm{C}$ for $10 \mathrm{sec}, 60^{\circ} \mathrm{C}$ for $20 \mathrm{sec}$, and $72^{\circ} \mathrm{C}$ for $30 \mathrm{sec}$. The primer sequences are listed in Table I and the $2^{-\Delta \Delta \mathrm{Cq}}$ method was used to analyze the level of mRNA (22).

Cells and cell culture. HeLa and 293T cells from the Cell Bank of the Chinese Academy of Sciences were cultured in Dulbecco's modified Eagle's medium (DMEM) with 4,500 mg/l glucose (Sigma-Aldrich; Merck KGaA) and 10\% fetal bovine serum (Biological Industries). Cell cultures were incubated in a humidified $5 \% \quad \mathrm{CO}_{2} / 95 \%$ air environment at $37^{\circ} \mathrm{C}$

Transfection. The $293 \mathrm{~T}$ cells with a high Neuritin expression were plated into 6 -well plates at $1 \times 10^{6}$ cells/well, and were then transfected with miR-199a mimics or a negative control (NC) mimic (Sangon Biotech Co., Ltd.) using Lipofectamine 3000 reagent (160 pM; Invitrogen; Thermo Fisher Scientific, Inc.). Following $12 \mathrm{~h}$ of incubation at $37^{\circ} \mathrm{C}$, the cells were collected for western blot and RT-qPCR analyses.

Western blot analysis. Neuritin protein levels in the 293T cells transfected with miR-199a and NC mimics and hippocampal tissues were detected by western blot analysis. Cells lysed in RIPA lysis buffer (Beyotime Institute of Biotechnology) with $1 \%$ protease inhibitor cocktail (Beyotime Institute of Biotechnology), centrifuged at $15,777 \mathrm{x} \mathrm{g}$ for $10 \mathrm{~min}$ at $4^{\circ} \mathrm{C}$, and the supernatant was collected. The amount of protein was determined using BCA protein assay kit (Beyotime Institute of Biotechnology). The sample volume of each well is $40 \mu \mathrm{g}$ for $293 \mathrm{~T}$ cells or $80-100 \mu \mathrm{g}$ for hippocampal tissues, respectively. Total protein was electrophoresed by $12.5 \%$ SDS-PAGE and transferred to PVDF membranes at $23 \mathrm{~V}$ for $43 \mathrm{~min}$. Membranes were blocked with Tris-buffered saline (TBS)/5\% fat-free skim milk for $2 \mathrm{~h}$ at room temperature, and then incubated with rabbit anti-Neuritin monoclonal antibody $\left(1: 1,000\right.$; ab64186, Abcam) for $16 \mathrm{~h}$ at $4^{\circ} \mathrm{C}$. The following day, the membranes were incubated with secondary goat anti-rabbit IgG-HRP antibody (1:2,500; ZB-2301, Zsgb-Bio) for $2 \mathrm{~h}$ at room temperature. Finally, proteins were detected using Clarity Western ECL substrates (Bio-Rad Laboratories, Inc.). $\beta$-actin (Zsgb-Bio) was used as an endogenous control. Adobe photoshop CC 2015 software was used for densitometry.

Luciferase reporter assay. HeLa cells were seeded in 24-well plates at $1 \times 10^{5}$ cells/well and co-transfected with NC mimics (Sangon Biotech Co., Ltd.) and pLUC-NC, NC mimics and pLUC-Nrn1 3'-UTR, miR-199a mimics (Sangon Biotech Co., Ltd.) and pLUC-Nrn1 3'-UTR, or miR-199a mimics and pLUC-Nrn1-mut 3'-UTR, with Lipofectamine 3000 (Invitrogen; Thermo Fisher Scientific, Inc.). After $12 \mathrm{~h}$, the cells were lysed, and their luciferase activity was measured using Dual-Luciferase reporter assay system and pLUC was 
Table I. Sequences of primers used for RT-qPCR.

\begin{tabular}{ll} 
Primer & \multicolumn{1}{c}{ Sequence } \\
\hline U6-F & 5'-CTCGCTTCGGCAGCACA-3' \\
U6-R & 5'-AACGCTTCACGAATTTGCGT-3' \\
miR-199a-5p RT & 5'-CTCAACTGGTGTCGTGGAGTCGGCAATTCAGTTGAGGAACAGGT-3' \\
miR-199a-5p F & 5'-ACACTCCAGCTGGGCCCAGTGTTCAGACT-3' \\
All R & 5'-TGGTGTCGTGGAGTCG-3' \\
Mouse Nrn1-F & 5'-ATTTCACTGATCCTCGCGGT-3' \\
Mouse Nrn1-R & 5'-CCAGTATGTGCACACGGTCT-3' \\
Mouse GAPDH-F & 5'-CAGGAGAGTGTTTCCTCGTCC-3' \\
Mouse GAPDH-R & 5'-TTCCCATTCTCGGCCTTGAC-3' \\
293T Nrn1-F & 5'-ATAGCGTATCTGGTGCAGGC-3' \\
293T Nrn1-R & 5' -TGTTCGTCTTGTCGTCCAGG-3' \\
293T $\beta-$-actin-F & 5'-GGGAAATCGTGCGTGACAT-3' \\
293T $\beta$-actin-R & 5'-GTCAGGCAGCTCGTAGCTCTT-3'
\end{tabular}

Nrn1, Neuritin; F, forward; R, reverse.

pmirGLO Dual-Luciferase miRNA Target Expression Vector (Promega Corporation). To normalize the reporter signal, the mean signal of the firefly luminescence was divided by the mean signal of Renilla luminescence.

Morris water maze. APP/PS1 and WT mice were transferred from the specific pathogen-free barrier to the behavioral laboratory to adapt to the environment for 7 days. The escape platform was located in a fixed spatial location $1 \mathrm{~cm}$ above the water surface on the 1st day, and was $1 \mathrm{~cm}$ below the water surface from the 2 nd to 5 th days. According to the circadian rhythm of the animals, the mice were placed into the water twice each night. The maximum trial length was $60 \mathrm{sec}$, and if a mouse did not reach the platform in the allotted time, they were manually guided to it. Upon reaching the invisible escape platform, the mice were left on it for an additional $5 \mathrm{sec}$ to allow for a survey of the spatial cues in the environment to guide future navigation to the platform. After each trial, the mice were wiped dry and left in the constant temperature heating station for $5 \mathrm{~min}$ to dry. Subsequently, they were placed back in their cages. Following 5 days of task acquisition, the probe test was performed over a period of $60 \mathrm{sec}$ during which the platform was removed to measure time and crossing times in the target quadrant. All trials were analyzed for latency, swim speed and swim path using AnyMaze (Clever Sys, Inc.) (23).

Statistical analysis. All experiments were performed in triplicate, and the results are presented as the means \pm standard error. Statistical analyses were performed using $\mathrm{R}$ software (RStudio3.6.2, https://www.r-project.org/), with $\mathrm{P}<0.05$ being set for statistical significance using Student-Newman-Keuls test.

\section{Results}

Cluster analysis of DEmiRNAs in AD. The miRNA data was derived from samples of patients with AD and AD model mice.
The 5 miRNA expression datasets (GSE48552, GSE46579, GSE46131, GSE16759 and GSE48028) and data from the literature were enrolled (Table II). First, quality control of the 5 datasets was conducted to normalize the measurement data between different samples using the $\mathrm{R}$ conductor package (Fig. S1). Second, the miRNAs in GSE48552, GSE46579, GSE46131, GSE16759 and GSE48028 were subjected to cluster analysis (Fig. 1A-E). Third, DEmiRNAs were screened using the cut-off criteria of P-value $<0.05$ and $\log \mathrm{FCl} \geq 1$ by the LIMMA R package (Fig. 1F-J). After analyzing the 5 miRNA expression datasets, 384 DEmiRNAs were screened (Table SI); another 323 DEmiRNAs were screened from the literature with the full miRNA expression profiles of Tg2576 AD model mice (21). In total, 707 DEmiRNAs were analyzed in $\mathrm{AD}$.

Screening candidate miRNAs. First, 1,348 miRNAs that could target the 3'-UTR of Neuritin (or Nrn1; Table SII) were predictively analyzed using 9 bioinformatics algorithms. Second, 36 miRNAs that overlapped in the databases of the 1,348 miRNAs that target Neuritin and the 707 DEmiRNAs in AD were screened (Fig. 2A). Third, 15 miRNAs with higher binding scores for Neuritin were selected (Table III), and the 10 candidate miRNAs with the highest AD/WT ratio among these were screened out (Fig. 2B). Additionally, GO enrichment analysis revealed that the candidate miRNAs were enriched in the nervous system as the biological process (BP) (Fig. 2C), were primarily distributed in the neuronal cell body, presynaptic membrane, neuron projection and postsynaptic density as the cellular component (CC) (Fig. 2D), and exhibited neurotrophin TRKA receptor binding and tau-protein kinase activity as the molecular function (MF) (Fig. 2E).

Detection of the candidate miRNAs in the hippocampus and cortex of APP/PS1 mice. To confirm the association between the candidate miRNAs and AD, the expression patterns of the candidate miRNAs in hippocampal and 


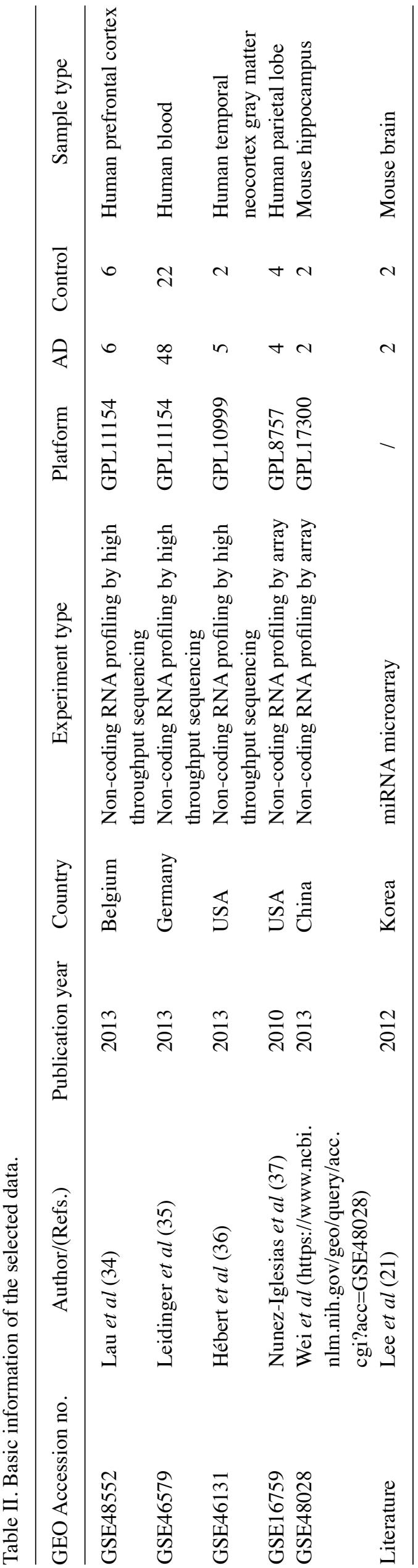

Table III. Ranking of the 36 miRNAs according to their Nrn1 binding scores.

\begin{tabular}{|c|c|}
\hline miRNAs & Binding scores (Nrn1) \\
\hline mmu-miR-199a-5p & 1 \\
\hline mmu-miR-151-5p & 1 \\
\hline mmu-miR-194-5p & 1 \\
\hline mmu-miR-342-3p & 1 \\
\hline mmu-miR-125a-5p & 1 \\
\hline mmu-miR-423-5p & 1 \\
\hline mmu-miR-770-3p & 1 \\
\hline mmu-miR-188-5p & 1 \\
\hline mmu-miR-690 & 1 \\
\hline mmu-miR-706 & 1 \\
\hline mmu-miR-331-3p & 0.92 \\
\hline mmu-miR-182-5p & 0.92 \\
\hline mmu-miR-125a-3p & 0.92 \\
\hline mmu-miR-139-3p & 0.92 \\
\hline mmu-miR-202-3p & 0.92 \\
\hline mmu-miR-129-5p & 0.92 \\
\hline mmu-miR-540-3p & 0.92 \\
\hline mmu-miR-540-5p & 0.92 \\
\hline mmu-miR-337-5p & 0.92 \\
\hline mmu-miR-709 & 0.92 \\
\hline mmu-miR-434-5p & 0.92 \\
\hline mmu-miR-409-5p & 0.92 \\
\hline mmu-miR-666-3p & 0.92 \\
\hline mmu-miR-338-5p & 0.92 \\
\hline mmu-miR-705 & 0.92 \\
\hline mmu-miR-671-5p & 0.92 \\
\hline mmu-miR-697 & 0.92 \\
\hline mmu-miR-34b-5p & 0.85 \\
\hline mmu-miR-324-5p & 0.85 \\
\hline mmu-miR-532-5p & 0.85 \\
\hline mmu-miR-296-5p & 0.85 \\
\hline mmu-miR-380-3p & 0.85 \\
\hline mmu-miR-574-5p & 0.85 \\
\hline mmu-miR-878-3p & 0.85 \\
\hline mmu-miR-96-5p & l \\
\hline mmu-miR-384-3p & / \\
\hline
\end{tabular}

cortical tissue from APP/PS1 (AD model) mice at 1, 4 and 7 months were examined. Compared with the WT mice, the levels of the candidate miRNAs were altered to varying degrees in the hippocampus of APP/PS1 mice (Table IV), among which, miR-199a upregulation was more significant at 4 months (Fig. 3A). In addition, the upregulated expression pattern of miR-199a remained slight altered at 1 month, peaked at 4 months, and was attenuated at 7 months (Fig. 3B).

Neuritin expression in the hippocampus and cortex of APP/PS1 mice. The neuritin mRNA levels were also measured in the hippocampal and cortical tissues of APP/PS1 and WT mice at 1, 4 and 7 months. Compared with the WT mice, the 


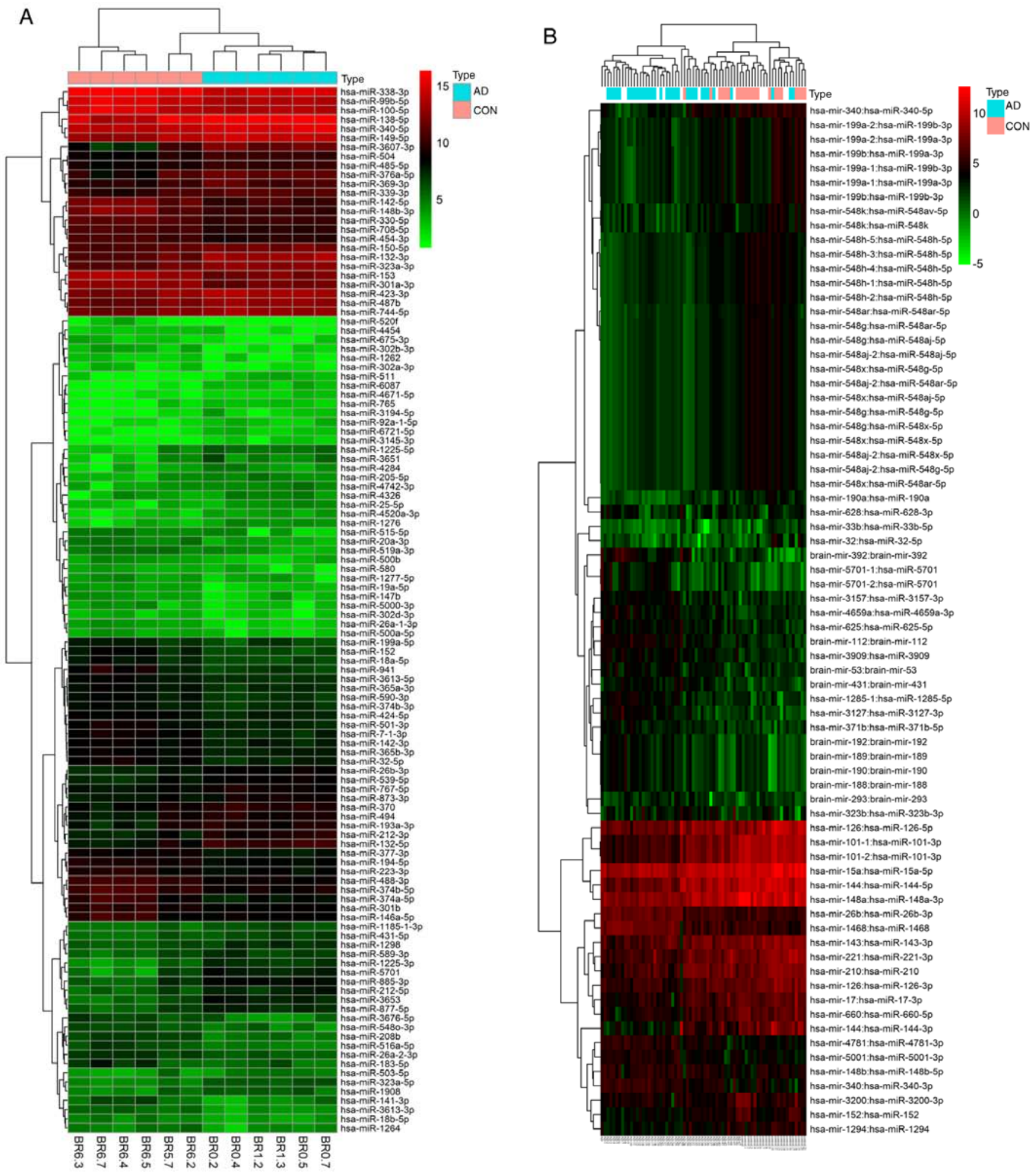

Figure 1. Cluster analysis of the miRNAs from 5 datasets. Heatmaps of DEmiRNAs from the (A) GSE48552 and (B) GSE46579 datasets. Each row represents a miRNA and each column represents a sample. The miRNA clustering tree is on the left, and the sample clustering tree appears at the top. The color scale shown at the top illustrates the relative expression level of miRNAs: Red indicates a high relative expression, while green indicates a low relative expression.

neuritin mRNA levels in the hippocampus of APP/PS1 mice were significantly decreased at 1,4 and 7 months (Fig. 4). Similarly, compared with WT mice, the Neuritin protein levels were significantly lower in the APP/PS1 mice at 4 and 7 months (Fig. 3D and E). Although Neuritin expression was not markedly altered at 1 month (Fig. 3C), it decreased significantly at 4 months, and exhibited a continued decline at 7 months (Fig. 3F).

miR-199a decreases Neuritin expression by targeting the Neuritin 3'-UTR. To examine the effects of miR-199a on Neuritin expression, miR-199a mimics were synthesized. 

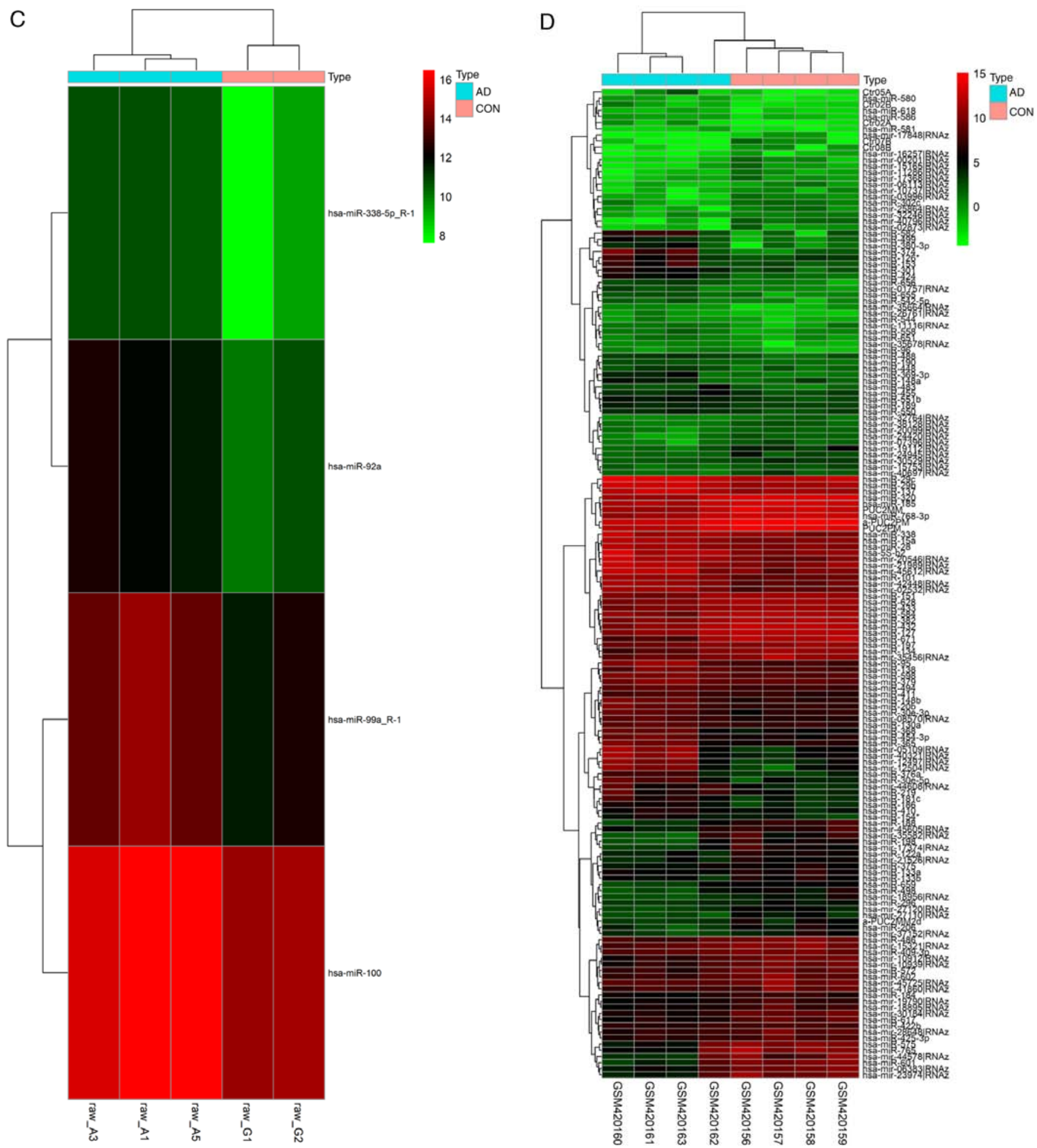

Figure 1. Continued. Cluster analysis of the miRNAs from 5 datasets. Heatmaps of DEmiRNAs from the (C) GSE46131 and (D) GSE416759 datasets. Each row represents a miRNA and each column represents a sample. The miRNA clustering tree is on the left, and the sample clustering tree appears at the top. The color scale shown at the top illustrates the relative expression level of miRNAs: Red indicates a high relative expression, while green indicates a low relative expression.

The results revealed that transfection with miR-199a mimics suppressed Neuritin mRNA (Fig. 5A) and protein (Fig. 5B) expression. The site within Nrn1 that was targeted by miR199a was predicted using bioinformatics software (Fig. 5C), and the pLUC-Nrn1 vector that contained the miR-199a targeting sites from the 3'-UTR of the Nrn1 transcript was constructed (Fig. 5D). It was observed that luciferase activity was decreased in the WT luciferase reporter system (pLUC-Nrn1); however, this decrease was reversed in the mutant luciferase reporter system (pLUC-Nrn1-mut) following stimulation with miRNA-199a mimic (Fig. 5E).

Evaluation of the spatial memory capacities of APP/PSI mice. The spatial memory of APP/PS1 mice was examined at 4 and 7 months using the Morris water maze test. The escape latency (Fig. 6A and B), swim speed (Fig. 6C and D), retention 


\section{$\mathrm{E}$}

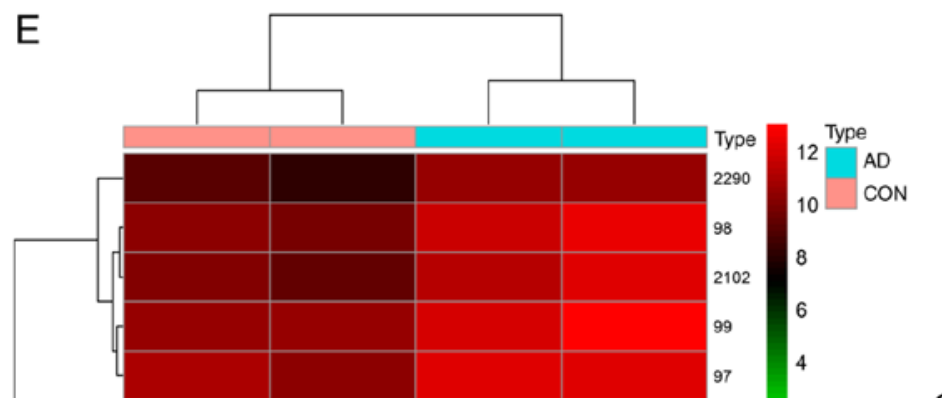

\section{G}

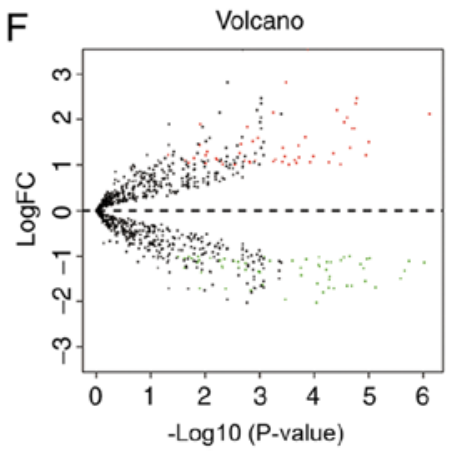

G Volcano

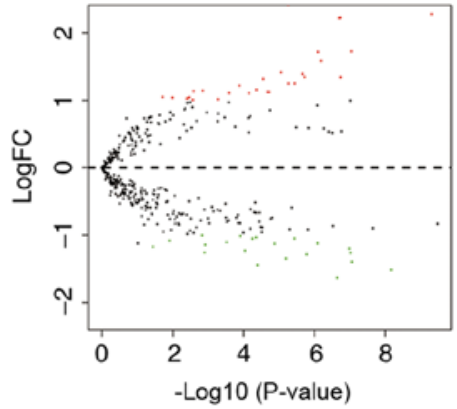

$\mathrm{H}$

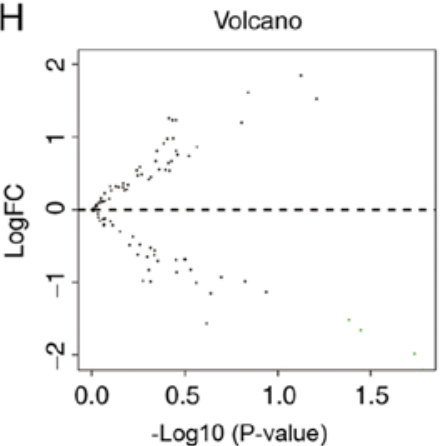

I

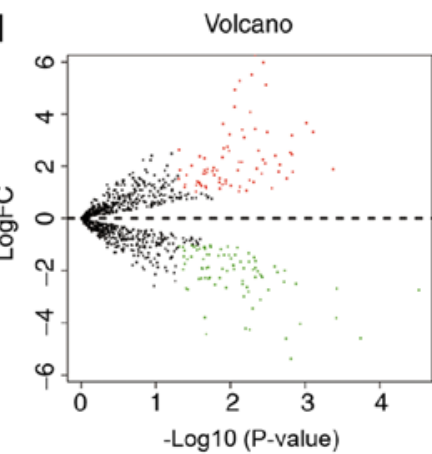

J

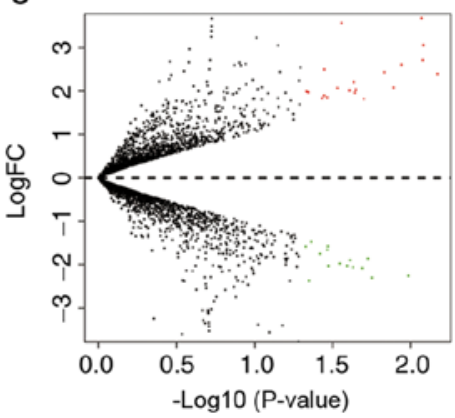

Figure 1. Continued. Cluster analysis of the miRNAs from 5 datasets. Heatmap of DEmiRNAs from the (E) GSE48028 dataset. Each row represents a miRNA and each column represents a sample. The miRNA clustering tree is on the left, and the sample clustering tree appears at the top. The color scale shown at the top illustrates the relative expression level of miRNAs: Red indicates a high relative expression, while green indicates a low relative expression. (F-J) Volcano plots of DEmiRNAs from the GSE48552, GSE46579, GSE46131, GSE416759 and GSE48028 datasets, respectively. 
A

Venn diagram selection of

the DEmiRNAs target neuritin in $A D$

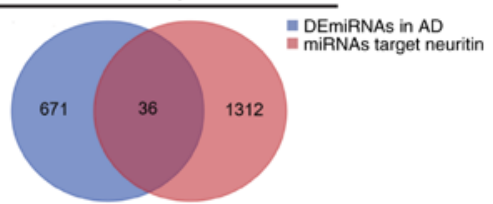

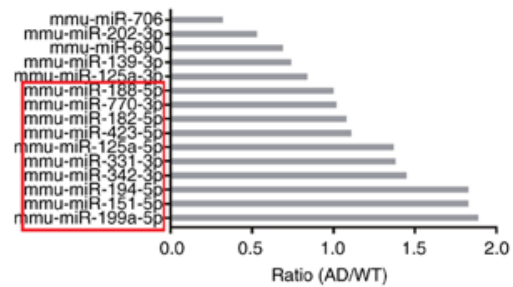

C

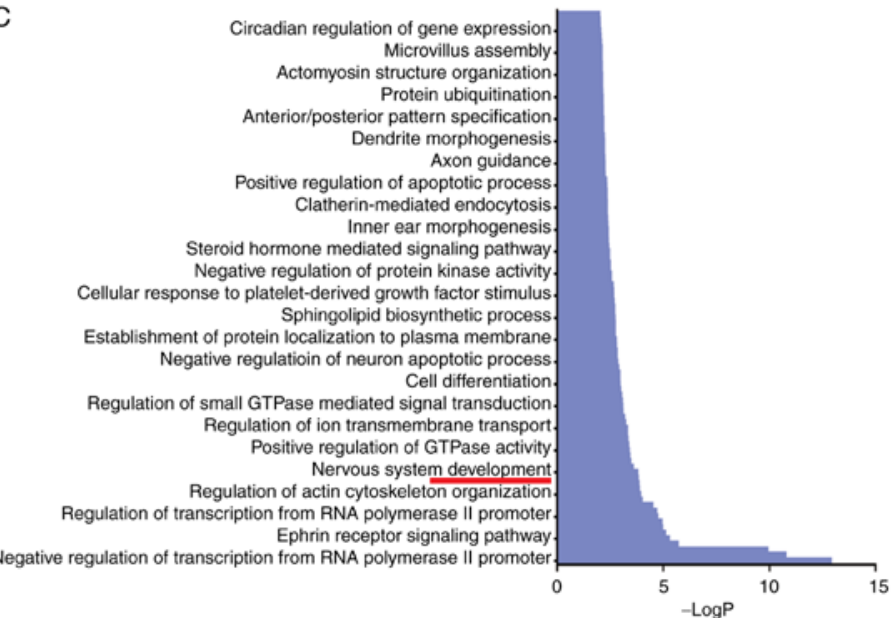

D Lateral plasma membrane

Synaptic vesicle

PML body

Clathrin-coated pit

Adaptor complex

Adaptor complex

Actin cyloskeleton

Growth cone

Cell projection

Heterochromatin

Nuclear lamina

Neuronal cell body

MLLT complex

Dendritic spine

Presynaptic membrane

cytoplasmic vesicle

Golgi membrane

Membrane

Nuclear matrix

Neuron projection

Transcription factor complex

Perinuclear region of cytoplasm

Postsynaptic density

Cell-cell junction

Cell-cell adherens junction

Focal adhesion

Cytoplasm.

$\underbrace{}_{0}$

2

Protein kinase inhibitor activity -

Peptide hormone binding

Neurotrophin TRKA receptor binding

amin binding

MRNA 3-UTR binding

SH3 domain binding

HMG box domain binding

Polyubiquitin binding

RNA polymerase II regulatory region sequence-specific DNA binding

Growth factor activity

Protein tyrosine/serine/threonine phosphatase activity
Insulin receptor binding

RNA polymerase II transcription factor activity, ligand-activated sequence-s

Kinesin binding

Transcriptional repressor activity, RNA polymerase II transcription regulato

Epidermal growth factor receptor binding

Histone acetyltransferase binding
Transcription factor binding

Protein homodimerization activity

beta-catenin binding

$\frac{\text { tau-protein kinase activity }}{\text { Protein binding }}$

Protein binding -

Figure 2. Screening of the candidate miRNAs. (A) Venn diagram for overlapping DEmiRNAs from AD (blue circle) and miRNAs that target Nrn1 (red circle) (B) A total of 10 candidate miRNAs with higher AD/WT ratios and high Neuritin binding are indicated within the red box. (C-E) The GO enrichment plots of candidate miRNA-targeted genes for biological process (BP), cellular component (CC) and molecular Function (MF). DEmiRNAs, differentially expressed miRNAs; AD, Alzheimer's disease; GO, Gene Ontology. 
Table IV. Detection of candidate miRNAs in the hippocampus and cortex of APP/PS1 mice.

\begin{tabular}{|c|c|c|c|c|c|c|}
\hline \multirow[b]{2}{*}{ miRNA/U6 $\left(x 10^{-4}\right)$} & \multicolumn{3}{|c|}{ Hippocampus } & \multicolumn{3}{|c|}{ Cortex } \\
\hline & 1 month & 4 months & 7 months & 1 month & 4 months & 7 months \\
\hline \multicolumn{7}{|l|}{ miR-199a-5p } \\
\hline WT & $0.88 \pm 0.24$ & $0.43 \pm 0.10$ & $0.64 \pm 0.12$ & $0.42 \pm 0.15$ & $0.46 \pm 0.06$ & $0.25 \pm 0.02$ \\
\hline APP/PS1 & $0.64 \pm 0.17$ & $1.26 \pm 0.08^{\mathrm{a}}$ & $0.62 \pm 0.18$ & $0.40 \pm 0.34$ & $0.55 \pm 0.16$ & $0.37 \pm 0.05^{\mathrm{a}}$ \\
\hline \multicolumn{7}{|l|}{ miR-151-5p } \\
\hline WT & $25.07 \pm 2.90$ & $26.38 \pm 3.82$ & $30.81 \pm 4.26$ & $21.96 \pm 7.51$ & $21.30 \pm 3.37$ & $15.75 \pm 2.03$ \\
\hline APP/PS1 & $23.07 \pm 1.58$ & $32.23 \pm 4.67^{\mathrm{a}}$ & $30.44 \pm 3.25$ & $20.75 \pm 2.79$ & $20.66 \pm 2.02$ & $15.48 \pm 1.62$ \\
\hline \multicolumn{7}{|l|}{ miR-342-3p } \\
\hline WT & $6.44 \pm 2.51$ & $3.62 \pm 2.38$ & $9.93 \pm 1.31$ & $9.41 \pm 3.05$ & $3.41 \pm 2.17$ & $7.06 \pm 1.50$ \\
\hline APP/PS1 & $7.28 \pm 2.10$ & $9.22 \pm 3.11$ & $8.00 \pm 1.54$ & $9.12 \pm 3.20$ & $9.72 \pm 4.47$ & $6.42 \pm 1.87$ \\
\hline \multicolumn{7}{|l|}{ miR-331-3p } \\
\hline WT & $17.36 \pm 2.81$ & $16.76 \pm 1.59$ & $17.47 \pm 2.84$ & $11.35 \pm 1.33$ & $12.24 \pm 1.57$ & $83.29 \pm 1.48$ \\
\hline APP/PS1 & $17.40 \pm 2.43$ & $17.29 \pm 2.28$ & $17.11 \pm 2.01$ & $14.51 \pm 1.15^{\mathrm{a}}$ & $10.78 \pm 1.46$ & $91.85 \pm 2.97$ \\
\hline \multicolumn{7}{|l|}{ miR-423-5p } \\
\hline WT & $5.02 \pm 0.72$ & $5.33 \pm 0.152$ & $5.44 \pm 0.13$ & $6.72 \pm 0.42$ & $3.98 \pm 0.49$ & $3.58 \pm 0.76$ \\
\hline APP/PS1 & $6.03 \pm 0.66^{\mathrm{a}}$ & $6.34 \pm 0.72$ & $4.73 \pm 0.71$ & $3.63 \pm 0.66$ & $4.04 \pm 0.53$ & $3.35 \pm 0.64$ \\
\hline \multicolumn{7}{|l|}{ miR-182-5p } \\
\hline WT & $0.01 \pm 0.00$ & $0.03 \pm 0.01$ & $0.02 \pm 0.01$ & $0.01 \pm 0.00$ & $0.11 \pm 0.04$ & $0.02 \pm 0.01$ \\
\hline APP/PS1 & $0.01 \pm 0.00$ & $0.05 \pm 0.01^{\mathrm{a}}$ & $0.04 \pm 0.01^{\mathrm{a}}$ & $0.01 \pm 0.01$ & $0.05 \pm 0.03$ & $1.89 \pm 0.01$ \\
\hline \multicolumn{7}{|l|}{ miR-188-5p } \\
\hline WT & $0.97 \pm 0.20$ & $0.64 \pm 0.11$ & $0.78 \pm 0.14$ & $0.48 \pm 0.14$ & $0.44 \pm 0.07$ & $0.30 \pm 0.09$ \\
\hline APP/PS1 & $1.01 \pm 0.21$ & $1.05 \pm 0.11^{\mathrm{a}}$ & $0.90 \pm 0.12$ & $0.58 \pm 0.12$ & $0.61 \pm 0.11^{\mathrm{a}}$ & $0.43 \pm 0.07^{\mathrm{a}}$ \\
\hline \multicolumn{7}{|l|}{ miR-770-3p } \\
\hline WT & $3.89 \pm 2.97$ & $3.11 \pm 0.47$ & $2.90 \pm 0.99$ & $3.68 \pm 1.85$ & $2.45 \pm 0.64$ & $4.57 \pm 3.05$ \\
\hline APP/PS1 & $3.88 \pm 1.73$ & $2.43 \pm 0.55$ & $2.72 \pm 0.57$ & $2.29 \pm 1.04$ & $1.94 \pm 0.46$ & $1.64 \pm 0.45$ \\
\hline \multicolumn{7}{|l|}{ miR-194-5p } \\
\hline WT & $6.88 \pm 1.06$ & $5.81 \pm 0.78$ & $6.46 \pm 0.82$ & $5.06 \pm 0.15$ & $4.51 \pm 0.75$ & $2.99 \pm 0.39$ \\
\hline APP/PS1 & $6.03 \pm 0.88$ & $6.34 \pm 1.07$ & $6.05 \pm 0.42$ & $5.37 \pm 1.10$ & $4.13 \pm 0.9 .9$ & $3.55 \pm 0.79$ \\
\hline \multicolumn{7}{|l|}{$m i R-125 a-5 p$} \\
\hline WT & $23.35 \pm 3.30$ & $44.43 \pm 7.73$ & $42.06 \pm 7.13$ & $22.44 \pm 4.55$ & $29.82 \pm 3.25$ & $29.03 \pm 3.41$ \\
\hline APP/PS1 & $24.11 \pm 3.72$ & $38.95 \pm 6.06$ & $43.24 \pm 6.76$ & $21.89 \pm 5.34$ & $27.17 \pm 3.35$ & $29.36 \pm 2.74$ \\
\hline
\end{tabular}

In the hippocampus and cortex of 1-, 4-, 7-month-old APP/PS1 and WT mice (n=3/group), the levels of the 10 candidate miRNAs were assessed by RT-qPCR. All data are normalized to U6 levels. Data are expressed as the means \pm SD. Significance was determined using a t-test for the data from the APP/PS1 and WT mice. ${ }^{\mathrm{P}} \mathrm{P}<0.05 \mathrm{vs}$. WT mice. WT, wild-type.

time and crossing times in the target quadrant (Fig. 6E and F) were calculated. According to the swimming speed of the mice, differences in the test indicators that were caused by the different exercise abilities of the mice were excluded. It was found that the spatial memory capacity of the APP/PS1 mice began to decrease at 4 months compared with the WT mice (Fig. 6E and G). Moreover, the spatial memory capacities of the 7-month-old APP/PS1 mice were significantly decreased compared with those of the WT mice (Fig. 6B, F and H).

\section{Discussion}

Neuritin is a neurotrophic factor that plays multiple roles, including roles in neurite growth, synaptic maturation and the maintenance of neuron survival, and also mediates neural development and neuroplasticity $(2-5,24-27)$. It has been found that Neuritin expression is decreased in mice with AD, and that Neuritin treatment can attenuate the synaptic deficits of AD mice $(13,14)$. However, the mechanisms responsible for the decreased expression of Neuritin in AD and whether miRNAs regulate Neuritin in AD remain unclear. In the present study, it was found that miR-199a decreased the Neuritin levels and was involved in the development of AD.

In the present study, after clustering the upregulated miRNAs in AD from the literature and datasets of AD using bioinformatics analysis, 707 DEmiRNAs were recruited (Table SI). Furthermore, the upregulated miRNAs that can target the 3'-UTR of Neuritin were predictively analyzed to 


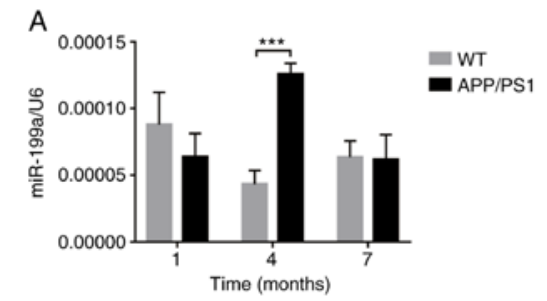

B

C

D

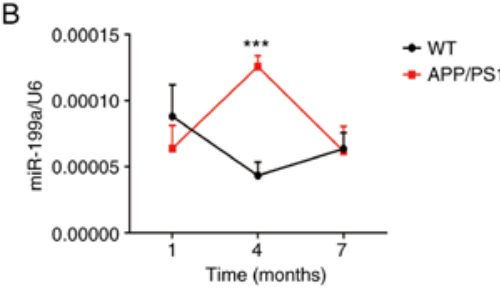

D 4 months

E 7 months
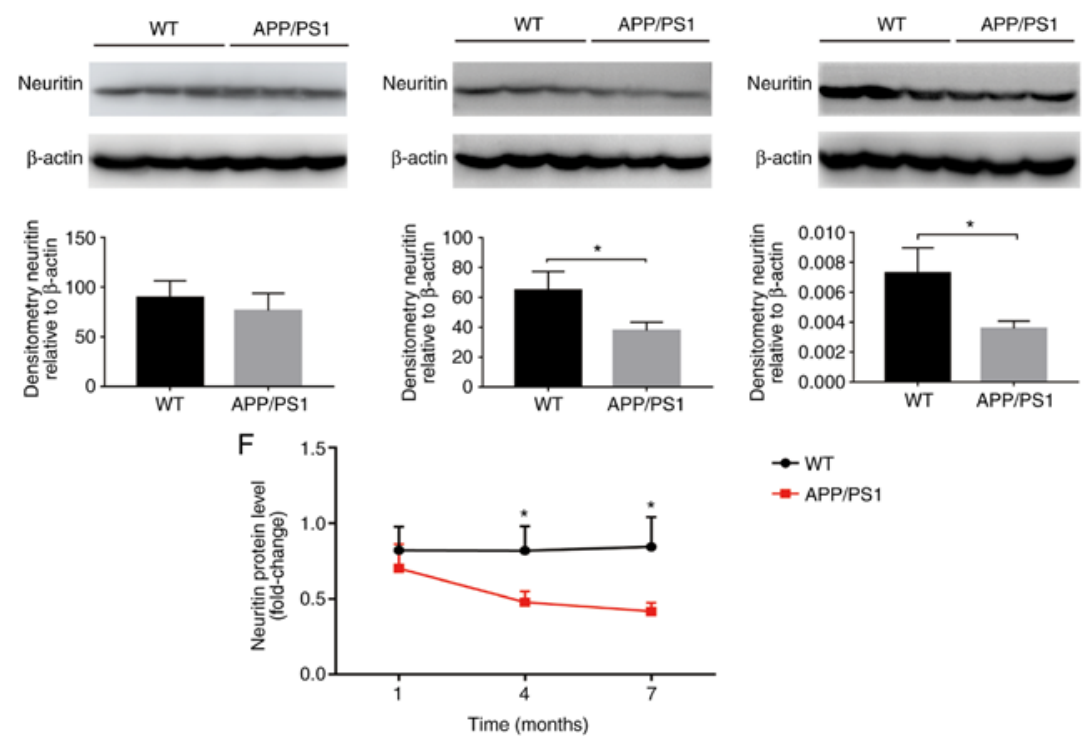

$\bullet W T$

- APP/PS1

Figure 3. Negative associatoin between miR-199a and Neuritin expression in the hippocampus of APP/PS1 mice. (A) miR-199a levels in the hippocampus of 1-, 4-, and 7-month-old mice were measured by RT-qPCR. (B) Line chart of miR-199a expression. (C-E) Neuritin protein expression in the hippocampus of $1-, 4-$, and 7-month-old mice (n=3/group) was analyzed by western blot analysis. (F) Line chart of Neuritin protein expression. All data are presented as the means \pm SD. ${ }^{*} \mathrm{P}<0.05,{ }^{* * * *} \mathrm{P}<0.001$ vs. WT. WT, wild-type.
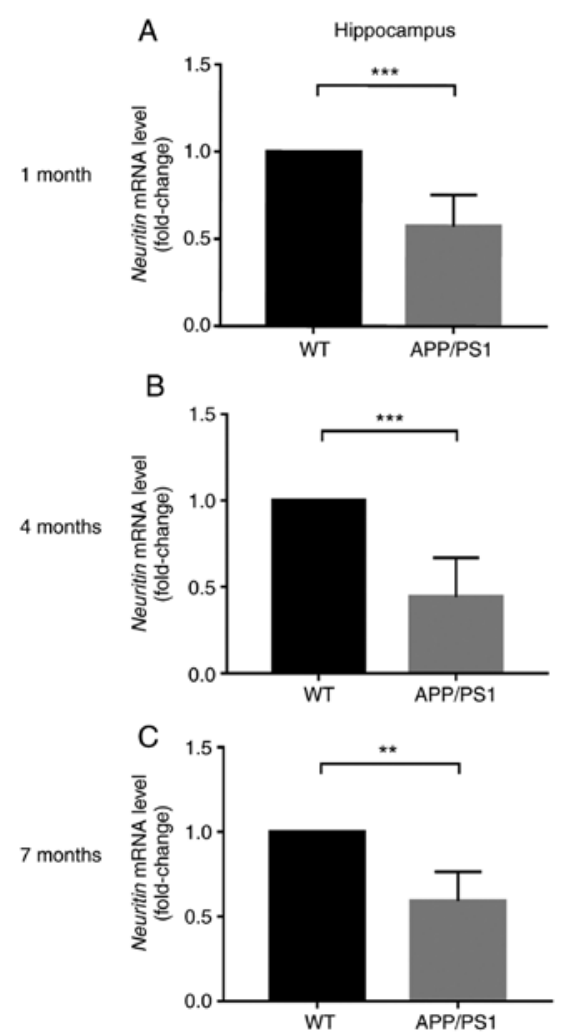

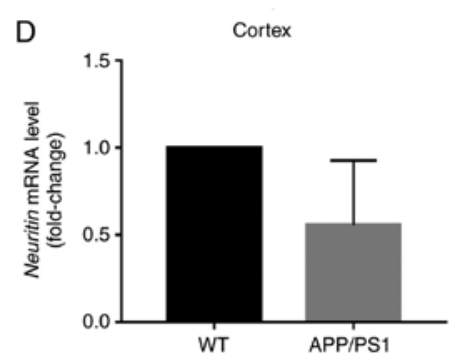

E
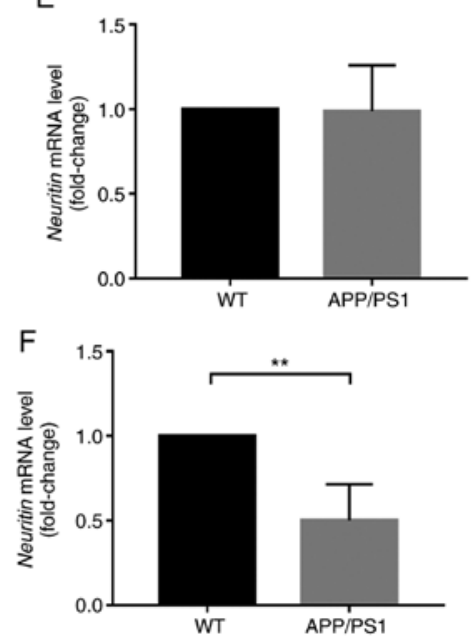

Figure 4. Neuritin expression in the hippocampus and cortex of APP/PS1 mice. (A-C) Neuritin mRNA expression was assessed by RT-qPCR in the hippocampus of 1-, 4-, and 7-month-old mice (n=3/group). (D-F) Neuritin mRNA expression was assessed by RT-qPCR in the cortex of 1-, 4-, and 7-month-old mice ( $\mathrm{n}=3$ /group). All data were normalized to GAPDH. All data are presented as the means $\pm \mathrm{SD}$. ${ }^{* *} \mathrm{P}<0.01,{ }^{* * *} \mathrm{P}<0.001$ vs. WT mice. WT, wild-type. 

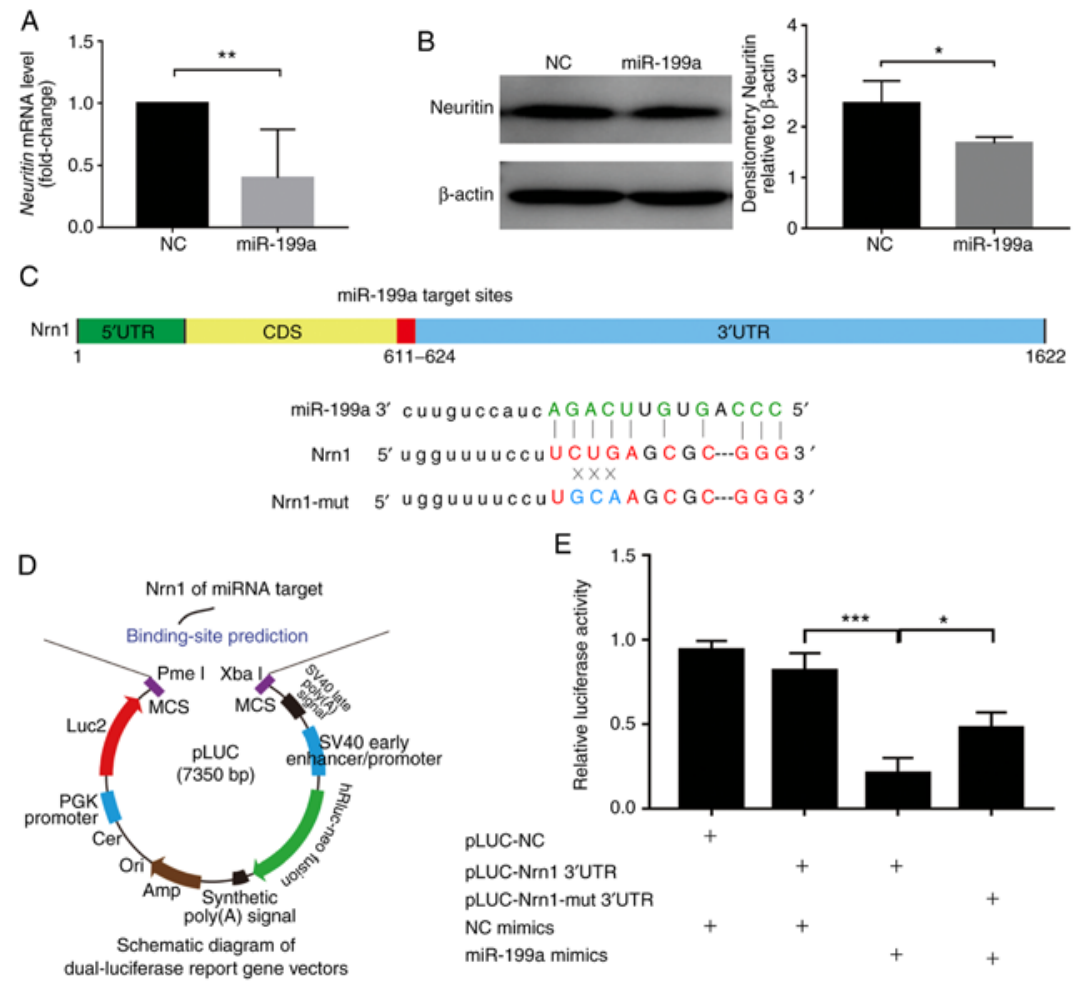

Figure 5. miR-199a regulates Neuritin expression. (A) Neuritin mRNA levels were detected by RT-qPCR in cells transfected with miR-199a mimics. (B) Neuritin protein levels were evaluated by western blot analysis in cells transfected with miR-199a mimics ( $\beta$-actin was used as the loading control). (C) Sites within the Nrn1 3'UTR that were predicted by bioinformatics software to be targeted by miR-199a. (D) The pLUC-Nrn1 vector contained the miR-199a site found in the Nrn1 3'UTR. (E) The luciferase activity from the Nrn1-mutated vector (pLUC-Nrn1-mut) and the Nrn1-wild-type vector (pLUC-Nrn1). Data in (A, B and E) are expressed as the means $\pm \mathrm{SD}(\mathrm{n}=3) .{ }^{*} \mathrm{P}<0.05,{ }^{* *} \mathrm{P}<0.01,{ }^{* * *} \mathrm{P}<0.001$ vs. control (blank) or NC mimics.

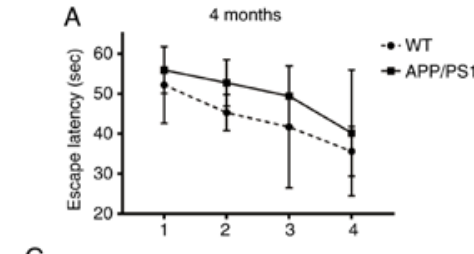

C

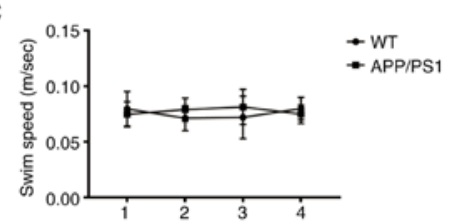

E

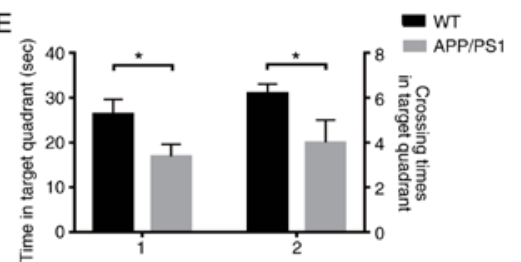

G

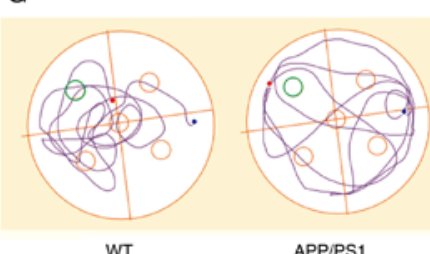

B
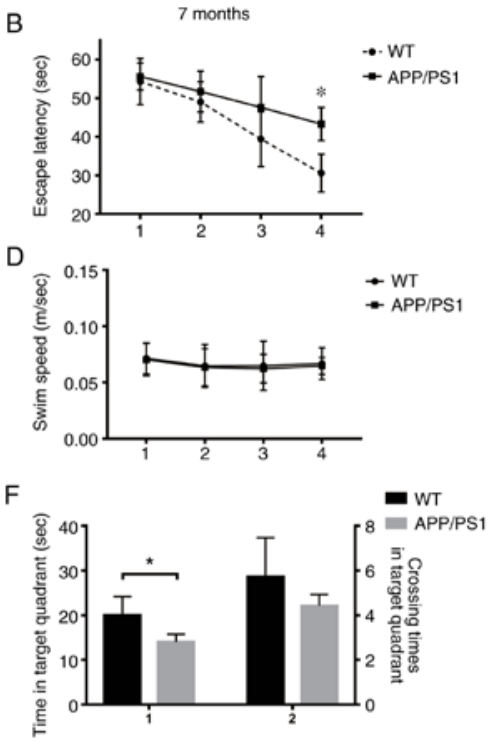

$\mathrm{H}$

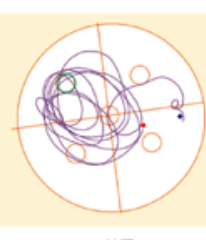

WT

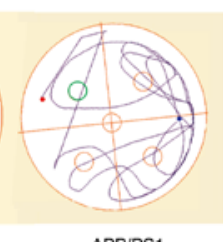

- Track start

Original plattorm

Figure 6. Evaluation of the spatial memory capacities of APP/PS1 mice. (A and B) The escape latency of mice was recorded at 4 and 7 months of age, respectively. (C and D) The swim speed of mice was recorded at 4 and 7 months of age, respectively. (E and F) Time required (left) and platform crossing (right) over the target quadrant of the original platform site were recorded to determine memory retention at 4 and 7 months of age, respectively. (G and $\mathrm{H}$ ) Swimming

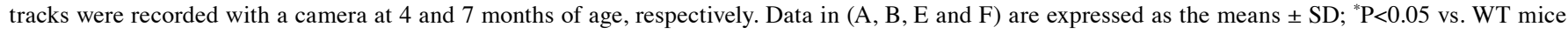
( $\mathrm{n}=7-10 \mathrm{mice} /$ group). WT, wild-type. 
screen out 36 miRNAs in AD that target Neuritin (Fig. 2A). Based on their binding scores (Table III), 10 candidate miRNAs with higher AD/WT ratios were selected for further analysis (Fig. 2B). Moreover, GO analysis on these 10 candidate miRNAs was performed, which revealed that they were enriched in the nervous system and were primarily distributed in the neuronal cell body, presynaptic membrane and neuron projection and postsynaptic density (Fig. 2C and D). Notably, the distribution patterns in the nervous system of the candidate miRNAs were consistent with those of Neuritin. Moreover, GO analysis revealed that the candidate miRNAs mediated molecular functions, such as neurotrophic protein TRKA receptor binding and tau kinase activity (Fig. 2E), indicating that the candidate miRNAs could be involved in the development of AD.

To further confirm that these candidate miRNAs target Neuritin from a bioinformatics perspective, the expression of the candidate miRNAs in hippocampal and cortical tissues from model mice with AD at different ages were detected. APP/PS1 mice are often used as models of $\mathrm{AD}$ as they have the major neuropathological features of $\mathrm{AD}$, including neuronal plaques in the hippocampus and cortex $(28,29)$ and neurofibrillary tangles (30,31). Compared with WT mice, the candidate miRNAs exhibited varying degrees of altered expression in the hippocampus of APP/PS1 mice (TableIV). Among the altered miRNAs, the changes in miR-199a expression were the most significant (Fig. 3A)

Neuritin expression was also detected in the hippocampus of APP/PS1 mice at 1, 4 and 7 months of age. The results revealed that Neuritin expression was significantly lower in the APP/PS1 mice compared with the WT mice at 4 and 7 months of age (Figs. 3C-E and 4A-C). Notably, the Neuritin expression pattern was opposite to that of miRNA-199a at 4 months (the early stages of AD). These results indicated that a high miR-199a expression was negatively associated with Neuritin expression both spatially and temporally in the hippocampus of APP/PS1 mice (Fig. 3B and F); thus, both may be involved in the development of AD.

To date, to the best of our knowledge there is no literature available on the regulation of Neuritin by miR-199a in AD; thus, it is unclear whether miR-199a can alter Neuritin expression. In the present study, using miR-199a mimics, it was found that miR-199a significantly decreased Neuritin levels (Fig. 5A and B). To verify that the decrease in Neuritin expression was due to direct miR-199a binding, luciferase reporter vectors containing the WT Neuritin 3'-UTR and a mutated construct were constructed. The results revealed that the decrease in luciferase activity was reversed after mutating only 3 bases in the Neuritin 3'-UTR (Fig. 5E). This suggested that miR-199a decreased the Neuritin levels by specifically binding to the Nrn1 3'-UTR.

Subsequently, the present study examined whether miR-199a is involved in the development of AD by regulating Neuritin expression. AD is a chronic degenerative nervous system disease characterized by progressive cognitive and memory impairments $(32,33)$. Therefore, the spatial memory of APP/PS1 mice was evaluated when miR-199a was altered. It was found that the spatial memory ability began to decrease at 4 months and was significantly decreased at 7 months in APP/PS1 mice (Fig. 6E and G). Of note, memory impairment occurred at the age of 4 months, when the miR-199a levels were at their highest. Memory loss was more pronounced at 7 months (Fig. 6B, F and H) when Neuritin expression decreased, suggesting that miR-199a-5p is involved in the development of AD by modulating Neuritin expression.

In the present study, evidence regarding the association between miR-199a and Neuritin in APP/PS1 mice was obtained through the integration of bioinformatics and molecular biology. It was found that miR-199a decreased the Neuritin levels by binding the Nrn1 3'-UTR. Finally, it was found that miR-199a was involved in the development of AD by regulating Neuritin expression. The findings of the present study provide a new perspective through which to better interpret the occurrence and development of AD.

\section{Acknowledgements}

The authors would like to thank the Animal Center of Hangzhou Normal University for breeding the mice. Dr Yanmei Tao (Institute of Life Sciences, College of Life and Environmental Sciences, Hangzhou Normal University, Hangzhou, China) kindly provided the Morris water maze test.

\section{Funding}

The present study was supported by the Natural Science Foundation of China (grant no. 81771173 to $\mathrm{JH}$ ) and the Zhejiang Provincial Natural Science Foundation of China (grant no. LY18H260002 to YH).

\section{Availability of data and materials}

The data used and/or analyzed during the present study are available from the corresponding author on reasonable request.

\section{Authors' contributions}

$\mathrm{JH}, \mathrm{JZ}$ and LY contributed to the conception or design of the study. DS contributed to the acquisition, analysis, or interpretation of all cell-level data. GL contributed to the acquisition, analysis, or interpretation of the AD mouse data. YH contributed to the acquisition, analysis and interpretation of the bioinformatics data. PZ contributed to the construction of the vectors. DS and JH contributed to the drafting of the the article and revising it critically for all content. All authors contributed to revising the work critically for important intellectual content.

\section{Ethics approval and consent to participate}

All experimental procedures were approved by the Animal Ethics Committee of Hangzhou Normal University.

\section{Patient consent for publication}

Not applicable.

\section{Competing interests}

The authors declare that they have no competing interests. 


\section{References}

1. Nedivi E, Hevroni D, Naot D, Israeli D and Citri Y: Numerous candidate plasticity-related genes revealed by differential cDNA cloning. Nature 363: 718-722, 1993.

2. Nedivi E, Wu GY and Cline HT: Promotion of dendritic growth by CPG15, an activity-induced signaling molecule. Science 281 1863-1866, 1998.

3. Naeve GS, Ramakrishnan M, Kramer R, Hevroni D, Citri Y and Theill LE: Neuritin: A gene induced by neural activity and neurotrophins that promotes neuritogenesis. Proc Natl Acad Sci USA 94: 2648-2653, 1997.

4. Cantallops I, Haas K and Cline HT: Postsynaptic CPG15 promotes synaptic maturation and presynaptic axon arbor elaboration in vivo. Nat Neurosci 3: 1004-1011, 2000.

5. Javaherian A and Cline HT: Coordinated motor neuron axon growth and neuromuscular synaptogenesis are promoted by CPG15 in vivo. Neuron 45: 505-512, 2005.

6. Karamoysoyli E, Burnand RC, Tomlinson DR and Gardiner NJ: Neuritin mediates nerve growth factor-induced axonal regeneration and is deficient in experimental diabetic neuropathy. Diabetes 57: 181-189, 2008.

7. Fargo KN, Alexander TD, Tanzer L, Poletti A and Jones KJ: Androgen regulates neuritin mRNA levels in an in vivo model of steroid-enhanced peripheral nerve regeneration. J Neurotrauma 25: 561-566, 2008

8. Zhao QR, Lu JM, Yao JJ,Zhang ZY, Ling C and Mei YA: Neuritin reverses deficits in murine novel object associative recognition memory caused by exposure to extremely low-frequency $(50 \mathrm{~Hz})$ electromagnetic fields. Sci Rep 5: 11768, 2015.

9. Zhang Y, Zhang S, Xian L, Tang J, Zhu J, Cui L, Li S, Yang L and Huang J: Expression and purification of recombinant human neuritin from Pichia pastoris and a partial analysis of its neurobiological activity in vitro. Appl Microbiol Biotechnol 99: 8035-8043, 2015.

10. Gao R, Li X, Xi S, Wang H, Zhang H, Zhu J, Shan L, Song X, Luo X, Yang L and Huang J: Exogenous Neuritin promotes nerve regeneration after acute spinal cord injury in rats. Hum Gene Ther 27: 544-554, 2016.

11. Wang H, Li X, Shan L, Zhu J, Chen R, Li Y, Yuan W, Yang L and Huang J: Recombinant hNeuritin promotes structural and functional recovery of sciatic nerve injury in rats. Front Neurosci 10: 589, 2016.

12. Scheltens P, Blennow K, Breteler MM, de Strooper B, Frisoni GB, Salloway S and Van der Flier WM: Alzheimer's disease. Lancet 388: 505-517, 2016.

13. Choi Y, Lee K, Ryu J, Kim HG, Jeong AY, Woo RS, Lee JH, Hyun JW, Hahn S, Kim JH and Kim HS: Neuritin attenuates cognitive function impairments in tg 2576 mouse model of Alzheimer's disease. PLoS One 9: e104121, 2014.

14. An K, Jung JH, Jeong AY, Kim HG, Jung SY, Lee K, Kim HJ, Kim SJ, Jeong TY, Son Y, et al: Neuritin can normalize neura deficits of Alzheimer's disease. Cell Death Dis 5: e1523, 2014.

15. Lewis BP, Burge CB and Bartel DP: Conserved seed pairing, often flanked by adenosines, indicates that thousands of human genes are microRNA targets. Cell 120: 15-20, 2005.

16. Filipowicz W, Bhattacharyya SN and Sonenberg N: Mechanisms of post-transcriptional regulation by microRNAs: Are the answers in sight? Nat Rev Genet 9: 102-114, 2008.

17. Chang TC and Mendell JT: MicroRNAs in vertebrate physiology and human disease. Annu Rev Genomics Hum Genet 8: 215-239, 2007.

18. Li F, Wei G, Bai Y, Li Y, Huang F, Lin J, Hou Q, Deng R, Zhou JH, Zhang SX and Chen DF: MicroRNA-574 is involved in cognitive impairment in 5-month-old APP/PS1 mice through regulation of neuritin. Brain Res 1627: 177-188, 2015.

19. Gao R, Wang L, Sun J, Nie K, Jian H, Gao L, Liao X, Zhang H, Huang J and Gan S: MiR-204 promotes apoptosis in oxidative stress-induced rat Schwann cells by suppressing neuritin expression. FEBS Lett 588: 3225-3232, 2014.
20. Shen NN, Zhang ZL, Li Z, Zhang C, Li H, Wang JL, Wang J and $\mathrm{Gu} \mathrm{ZC}$ : Identification of microRNA biomarkers in atrial fibrillation: A protocol for systematic review and bioinformatics analysis. Medicine (Baltimore) 98: e16538, 2019.

21. Lee ST, Chu K, Jung KH, Kim JH, Huh JY, Yoon H, Park DK, Lim JY, Kim JM, Jeon D, et al: MiR-206 regulates brain-derived neurotrophic factor in Alzheimer disease model. Ann Neurol 72: 269-277, 2012.

22. Livak KJ and Schmittgen TD: Analysis of relative gene expression data using real-time quantitative PCR and the 2(-Delta Delta C(T)) method. Methods 25: 402-408, 2001.

23. Morris R: Developments of a water-maze procedure for studying spatial learning in the rat. J Neurosci Methods 11: 47-60, 1984.

24. Putz U, Harwell C and Nedivi E: Soluble CPG15 expressed during early development rescues cortical progenitors from apoptosis. Nat Neurosci 8: 322-331, 2005.

25. Fujino T, Leslie JH, Eavri R, Chen JL, Lin WC, Flanders GH, Borok E, Horvath TL and Nedivi E: CPG15 regulates synapse stability in the developing and adult brain. Genes Dev 25: 2674-2685, 2011

26. Corriveau RA, Shatz CJ and Nedivi E: Dynamic regulation of cpg15 during activity-dependent synaptic development in the mammalian visual system. J Neurosci 19: 7999-8008, 1999.

27. Fujino T, Wu Z, Lin WC, Phillips MA and Nedivi E: cpg15 and cpg15-2 constitute a family of activity-regulated ligands expressed differentially in the nervous system to promote neurite growth and neuronal survival. J Comp Neurol 507: 1831-1845, 2008.

28. Hansra GK, Popov G, Banaczek PO, Vogiatzis M, Jegathees T, Goldbury CS and Cullen KM: The neuritic plaque in Alzheimer's disease: Perivascular degeneration of neuronal processes. Neurobiol Aging 82: 88-101, 2019.

29. Gouras GK, Olsson TT and Hansson O: $\beta$-Amyloid peptides and amyloid plaques in Alzheimer's disease. Neurotherapeutics 12: 3-11, 2015.

30. Theofilas P, Ehrenberg AJ, Nguy A, Thackrey JM, Dunlop S, Mejia MB, Alho AT, Paraizo Leite RE, Rodriguez RD, Suemoto CK, et al: Probing the correlation of neuronal loss, neurofibrillary tangles, and cell death markers across the Alzheimer's disease Braak stages: A quantitative study in humans. Neurobiol Aging 61: 1-12, 2018.

31. Lacosta AM, Insua D, Badi H, Pesini P and Sarasa M: Neurofibrillary tangles of A $\beta \mathrm{x}-40$ in Alzheimer's disease brains. J Alzheimers Dis 58: 661-667, 2017.

32. Fainstein N, Dan-Goor N and Ben-Hur T: Resident brain neural precursor cells develop age-dependent loss of therapeutic functions in Alzheimer's mice. Neurobiol Aging 72: 40-52, 2018.

33. Friedman E, Lerer B and Kuster J: Loss of cholinergic neurons in the rat neocortex produces deficits in passive avoidance learning. Pharmacol Biochem Behav 19: 309-312, 1983.

34. Lau P, Bossers K, Janky R, Salta E, Frigerio CS, Barbash S, Rothman R, Sierksma AS, Thathiah A, Greenberg D, et al: Alteration of the microRNA network during the progression of Alzheimer's disease. EMBO Mol Med 5: 1613-1634, 2013.

35. Leidinger P, Backes C, Deutscher S, Schmitt K, Mueller SC, Frese K, Haas J, Ruprecht K, Paul F, Stähler C, et al: A blood based 12-miRNA signature of Alzheimer disease patients. Genome Biol 14: R78, 2013.

36. Hébert SS, Wang WX, Zhu Q and Nelson PT: A study of small RNAs from cerebral neocortex of pathology-verified Alzheimer's disease, dementia with lewy bodies, hippocampal sclerosis, frontotemporal lobar dementia, and non-demented human controls. J Alzheimers Dis 35: 335-348, 2013.

37. Nunez-Iglesias J, Liu CC, Morgan TE, Finch CE and Zhou XJ: Joint genome-wide profiling of miRNA and mRNA expression in Alzheimer's disease cortex reveals altered miRNA regulation. PLoS One 5: e8898, 2010.

This work is licensed under a Creative Commons Attribution-NonCommercial-NoDerivatives 4.0 International (CC BY-NC-ND 4.0) License. 